\title{
Fractional order of Legendre-type matrix polynomials
}

\author{
M. Zayed ${ }^{1}$, M. Hidan' ${ }^{1}$ M. Abdalla ${ }^{1,2^{*}}$ (1) and M. Abul-Ez ${ }^{3}$
}

\section{"Correspondence:}

moabdalla@kku.edu.sa

'Department of Mathematics, College of Science, King Khalid University, P.O. Box 9004, 61413, Abha, Saudi Arabia

${ }^{2}$ Department of Mathematics, Faculty of Science, South Valley University, 83523 Qena, Egypt Full list of author information is available at the end of the article

\section{Springer}

\begin{abstract}
Recently, special functions of fractional order calculus have had many applications in various areas of mathematical analysis, physics, probability theory, optimization theory, graph theory, control systems, earth sciences, and engineering. Very recently, Zayed et al. (Mathematics 8:136, 2020) introduced the shifted Legendre-type matrix polynomials of arbitrary fractional orders and their various applications utilizing Rodrigues matrix formulas. In this line of research, we use the fractional order of Rodrigues formula to provide further investigation on such Legendre polynomials from a different point of view. Some properties, such as hypergeometric representations, continuation properties, recurrence relations, and differential equations, are derived. Moreover, Laplace's first integral form and orthogonality are obtained.
\end{abstract}

MSC: $15 \mathrm{~A} 15 ; 33 \mathrm{C} 70 ; 33 \mathrm{C} 05 ; 33 \mathrm{D} 15$

Keywords: Special matrix functions; Fractional calculus; Legendre matrix polynomials; Rodrigues-type formula

\section{Introduction}

The recent advances in fractional order calculus (FOC) are dominated by its multidisciplinary applications. Undoubtedly, fractional calculus has become an exciting new mathematical approach to solving various problems in mathematics, model physical, engineering, and many branches of science (see, for example, [2-7] and the references therein). The "special functions of fractional order calculus" (SF of FOC) as generalized fractional calculus operators of some classical special functions were found by Kiryakova $[8,9]$ and Agarwal [2]. Recently, there has been an increasing interest to use classes of special functions, referred to as (SF of FOC), that play most important role in the theory of differentiation and integration of arbitrary order (i.e. FC) and appear as solutions of fractional order differential equations and systems (see e.g. [10-15]).

On the contrary, the mainstream and, perhaps, the most effective approach to the field of special functions of matrix argument is the fractional calculus approach, recently presented in the fundamental works (for instance, see [16-19]). Analogous to the classical case, it is also noticed that Rodrigues matrix formula is a useful approach to define a sequence of orthogonal matrix polynomials (see [1, 20-25]). This approach allows estab-

(c) The Author(s) 2020. This article is licensed under a Creative Commons Attribution 4.0 International License, which permits use sharing, adaptation, distribution and reproduction in any medium or format, as long as you give appropriate credit to the original author(s) and the source, provide a link to the Creative Commons licence, and indicate if changes were made. The images or other third party material in this article are included in the article's Creative Commons licence, unless indicated otherwise in a credit line to the material. If material is not included in the article's Creative Commons licence and your intended use is not permitted by statutory regulation or exceeds the permitted use, you will need to obtain permission directly from the copyright holder. To view a copy of this licence, visit http://creativecommons.org/licenses/by/4.0/. 
lishing many interesting properties of the matrix polynomials. Moreover, these generalized matrix formulas allow to define new classes of special matrix functions and matrix polynomials and to include fractional order differentiation.

The Legendre polynomials are used by mathematicians and engineers for a variety of mathematical and numerical solutions. For example, Legendre and associate Legendre polynomials are widely used in the determination of wave functions of electrons in the orbits of an atom [26] and in the determination of potential functions in the spherically symmetric geometry [27], etc. The Legendre polynomials are applicable in fluid dynamics to study the flow around the outside of a puff of hot gas rising through the air, see for details $[28,29]$. For readers, they can find other applications of these polynomials in [3035]. Later on, a new kind of the Legendre matrix polynomials was introduced by Upadhyaya and Shehata $[36,37]$. Within the frame, they discussed some fundamental properties and applications of these matrix polynomials. Motivated by certain recent extensions of the shifted Legendre-type matrix polynomials of arbitrary fractional orders utilizing Rodrigues matrix formulas [1], we introduce and examine some properties of the fractional order of Legendre-type matrix polynomials generated by fractional analogues of the Rodrigues-type formulas of these polynomials. The paper is organized, as follows: Sect. 2 includes the basic definitions and properties used in the following sections. In Sect. 3, we define the Legendre-type matrix polynomials of arbitrary fractional orders. The section also includes the hypergeometric matrix representations and establish that these Legendre-type matrix polynomials are continuous functions of arbitrary fractional orders. Recurrence relations and development of second-order matrix differential equations satisfied by these polynomials are provided in Sect. 4. Laplace's first integral form and the orthogonality of Legendre-type matrix polynomials of arbitrary fractional orders are proved in Sect. 5. We reach the conclusion in Sect. 6.

\section{Preliminaries}

In this section, we recall some definitions and terminologies which will be used to prove the main results in the next sections.

Definition 2.1 A matrix $D$, in the space of complex matrices $\mathbb{C}^{N \times N}$, is called positive stable [25] if

$$
\operatorname{Re}(z)>0, \quad z \in \sigma(D)
$$

where $\sigma(D)$ is the spectrum of matrix $D$.

Definition 2.2 Let $D$ be a positive stable matrix in $\mathbb{C}^{N \times N}$. The gamma matrix function $\Gamma(D)$ is defined in [25] as follows:

$$
\Gamma(D)=\int_{0}^{\infty} e^{-z} z^{D-I} d z ; \quad z^{D-I}=\exp ((D-I) \ln z) .
$$

Definition 2.3 ([25]) For all $D$ in $\mathbb{C}^{N \times N}$, we assume

$$
D+n I \text { is invertible for all } n \in \mathbb{N}_{0}:=\mathbb{N} \cup\{0\},
$$


and the Pochhammer symbol (the shifted factorial) is defined by

$$
(D)_{n}=D(D+I) \cdots(D+(n-1) I)=\Gamma(D+n I) \Gamma^{-1}(D) ; \quad(D)_{0} \equiv I
$$

where $I$ is an identity matrix in $\mathbb{C}^{N \times N}$.

Lemma 2.1 (Lancaster [38]) Let D be a matrix in $\mathbb{C}^{N \times N}$ such that $\|D\|<1$ and $\|I\|=1$, then $(I+D)^{-1}$ exists, and we have

$$
(I+D)^{-1}=I-D+D^{2}-D^{3}+D^{4}-D^{5}+\cdots,
$$

where $\|D\|$ is denoted by

$$
\|D\|=\sup _{x \neq 0} \frac{\|D x\|_{2}}{\|x\|_{2}}
$$

for a vector $y$ in $\mathbb{C}^{N},\|y\|_{2}=\left(y^{H} y\right)^{\frac{1}{2}}$ is the Euclidean norm of $y$, and $y^{H}$ denotes the Hermitian adjoint of $y$.

Definition 2.4 ([24, 25]) Let $s$ and $r$ be finite positive integers, the generalized hypergeometric matrix function is defined by the matrix power series

$$
{ }_{s} F_{r}(E ; D ; z)=\sum_{n=0}^{\infty} \prod_{i=1}^{s}\left(E_{i}\right)_{n} \prod_{j=1}^{r}\left[\left(D_{j}\right)_{n}\right]^{-1} \frac{z^{n}}{n !},
$$

where $E_{i}, 1 \leq i \leq s$, and $D_{j}, 1 \leq j \leq r$, are commutative matrices in $\mathbb{C}^{N \times N}$ and

$$
D_{j}+n I \text { are invertible for all integers } n \in \mathbb{N}_{0} \text {. }
$$

Note that for $s=2, r=1$ we get the Gauss hypergeometric matrix function ${ }_{2} F_{1}$ (see [25]).

Definition 2.5 ([2, 8, 9]) The fractional order integral (Riemann-Liouville operator) of the function $f(u)$ of order $\alpha$ is defined by

$$
\left(I_{a}^{\alpha} f\right)(u)=\frac{1}{\Gamma(\alpha)} \int_{a}^{\infty}(u-t)^{\alpha-1} f(t) d t
$$

where $a \in \mathbb{R}$ and $\alpha \in \mathbb{R}^{+}$.

Definition 2.6 ([1, 17]) Let $D$ be a positive stable matrix in $\mathbb{C}^{N \times N}$ and $\mu \in \mathbb{C}$ such that $\operatorname{Re}(\mu)>0$, the Riemann-Liouville fractional integral of order $\mu$ is defined as follows:

$$
\mathbf{I}^{\mu}\left(\zeta^{D}\right)=\frac{1}{\Gamma(\mu)} \int_{0}^{\zeta}(\zeta-z)^{\mu-1} \zeta^{D} d z, \quad D \in \mathbb{C}^{N \times N},
$$

where $\operatorname{Re}(z)>0$. 
Lemma $2.2([1,18])$ Let $D$ be a positive stable matrix in $\mathbb{C}^{N \times N}$ such that $\operatorname{Re}(\mu)>0$. Then the Riemann-Liouville fractional integrals of order $\mu$ can be written as

$$
\mathbf{I}^{\mu}\left(\zeta^{D-I}\right)=\Gamma(D) \Gamma^{-1}(D+\mu I) \zeta^{D+(\mu-1) I} .
$$

For the fractional order derivative, we have the following definition.

Definition $2.7([2,9,39])$ The Liouville-Caputo fractional derivative of order $\alpha \in(n-$ $1, n)(n \in \mathbb{N}:=\{1,2, \ldots\})$ of $f(\tau), \tau \geq a$ is given by

$$
D_{a}^{\alpha} f(\tau)=I_{a}^{n-\alpha} D^{n} f(\tau), \quad D=\frac{d}{d \tau}
$$

This definition is more convenient in many applications in physics, engineering, and applied sciences [3, 9, 14, 15, 39]. Moreover, it generalizes (interpolates) the definition of integer order derivative. The fractional derivative of the product functions $g(\tau) f(\tau)$ is given by the Leibniz rule in the form (cf. $[2,15])$

$$
D^{\alpha}[g(\tau) f(\tau)]=\sum_{s=0}^{\infty}\left(\begin{array}{l}
\alpha \\
s
\end{array}\right) D^{s} g(\tau) D^{\alpha-s} f(\tau)
$$

\section{Fractional Legendre matrix polynomials and hypergeometric representation}

Following [25, 36, 37], we adapt the Legendre-type matrix polynomials to the fractional setting as follows.

Definition 3.1 (Fractional Legendre matrix polynomial) Let $E$ be a positive stable matrix in $\mathbb{C}^{N \times N}$, and let us denote $\sqrt{E}=E^{\frac{1}{2}}=\exp \left(\frac{1}{2} \log E\right)$ the image of the function $z^{\frac{1}{2}}=$ $\exp \left(\frac{1}{2} \log \eta\right)$ by the Riesz-Dunford functional calculus, acting on the matrix $E$, where $\log \eta$ denotes the principal branch of the complex logarithm (cf. $[25,40]$ ). We define the Legendre-type matrix functions (LTMFs) by the Rodrigues formula

$$
L_{\frac{1}{m}}(z, E)=\frac{1}{\Gamma\left(\frac{1}{m}+1\right)} D_{-1}^{\frac{1}{m}}(\sqrt{2 E})^{-\frac{1}{m}}\left\{\left(\frac{z \sqrt{2 E}}{2}\right)^{2}-I\right\}^{\frac{1}{m}}, \quad m=1,2,3, \ldots
$$

and, for $\alpha \in(-1,0)$, the Legendre-type matrix functions of order $\alpha$ are defined by

$$
L_{\alpha}(z, E)=\frac{1}{\Gamma(\alpha+1)}(\sqrt{2 E})^{-\alpha} I_{-1}^{-\alpha}\left[\left(\frac{z \sqrt{2 E}}{2}\right)^{2}-I\right]^{\alpha} .
$$

\subsection{Hypergeometric representation}

Theorem 3.1 The matrix polynomials $L_{\frac{1}{m}}(z, E)$ have the following representations in terms of the Gauss hypergeometric function:

(i) $L_{\frac{1}{m}}(z, E)=\left\{\frac{1}{2}\left(\frac{z \sqrt{2 E}}{2}-I\right)\right\}^{\frac{1}{m}}{ }_{2} F_{1}\left(-\frac{1}{m},-\frac{1}{m} ; 1 ;\left(\frac{z \sqrt{2 E}}{2}+I\right)\left(\frac{z \sqrt{2 E}}{2}-I\right)^{-1}\right)$.

(ii) $L_{\frac{1}{m}}(z, E)=(-1)^{\frac{1}{m}}{ }_{2} F_{1}\left(-\frac{1}{m}, \frac{1}{m}+1 ; 1 ; \frac{2 I+z \sqrt{2 E}}{4}\right)$.

(iii) $L_{\alpha}(z, E)=(-1)^{\alpha}{ }_{2} F_{1}\left(-\alpha, \alpha+1 ; 1 ; \frac{2 I+z \sqrt{2 E}}{4}\right) ; \alpha \in(-1,0)$. 
Proof (i) According to Definition 3.1, we can write

$$
L_{\frac{1}{m}}(z, E)=\frac{1}{\Gamma\left(\frac{1}{m}+1\right)} D_{-1}^{\frac{1}{m}}(\sqrt{2 E})^{-\frac{1}{m}}\left\{\frac{z \sqrt{2 E}}{2}-I\right\}^{\frac{1}{m}}\left\{\frac{z \sqrt{2 E}}{2}+I\right\}^{\frac{1}{m}} .
$$

Using the Leibniz rule for fractional derivative, we obtain

$$
\begin{aligned}
L_{\frac{1}{m}}(z, E)= & \frac{(\sqrt{2 E})^{-\frac{1}{m}}}{\Gamma\left(\frac{1}{m}+1\right)} \sum_{k=0}^{\infty} C_{k}^{\frac{1}{m}} D^{k}\left(\frac{z \sqrt{2 E}}{2}-I\right)^{\frac{1}{m}} D_{-1}^{\frac{1}{m}-k}\left(\frac{z \sqrt{2 A}}{2}+I\right)^{\frac{1}{m}} \\
= & \left\{\frac{1}{2}\left(\frac{z \sqrt{2 E}}{2}-I\right)\right\}^{\frac{1}{m}} \\
& \times \sum_{k=0}^{\infty} \frac{\left\{\Gamma\left(\frac{1}{m}+1\right)\right\}^{2}}{\left\{\Gamma\left(\frac{1}{m}-k+1\right)\right\}^{2}\{\Gamma(k+1)\}^{2}}\left(\frac{z \sqrt{2 E}}{2}+I\right)^{k}\left(\frac{z \sqrt{2 E}}{2}-I\right)^{-k} .
\end{aligned}
$$

Thus,

$$
L_{\frac{1}{m}}(z, E)=\left\{\frac{1}{2}\left(\frac{z \sqrt{2 E}}{2}-I\right)\right\}^{\frac{1}{m}}{ }_{2} F_{1}\left(-\frac{1}{m},-\frac{1}{m} ; 1 ;\left(\frac{z \sqrt{2 E}}{2}+I\right)\left(\frac{z \sqrt{2 E}}{2}-I\right)^{-1}\right) .
$$

(ii) Since

$$
\begin{aligned}
L_{\frac{1}{m}}(z, E) & =\frac{1}{\Gamma\left(\frac{1}{m}+1\right)} D_{-1}^{\frac{1}{m}}(\sqrt{2 E})^{-\frac{1}{m}}\left\{\left(\frac{z \sqrt{2 E}}{2}\right)^{2}-I\right\}^{\frac{1}{m}} \\
& =\frac{D_{-1}^{\frac{1}{m}}(\sqrt{2 E})^{-\frac{1}{m}}}{\Gamma\left(\frac{1}{m}+1\right)}\left\{(-1)^{\frac{1}{m}}\left(\frac{z \sqrt{2 E}}{2}+I\right)^{\frac{1}{m}}\left(I-\frac{x \sqrt{2 E}}{2}\right)^{\frac{1}{m}}\right\} \\
& =\frac{1}{\Gamma\left(\frac{1}{m}+1\right)} D_{-1}^{-\frac{1}{m}}(\sqrt{2 E})^{\frac{1}{m}}\left\{(-2)^{\frac{1}{m}}\left(\frac{z \sqrt{2 E}+2 I}{2}\right)^{\frac{1}{m}} \cdot\left(I-\frac{z \sqrt{2 E}+2 I}{4}\right)^{\frac{1}{m}}\right\} .
\end{aligned}
$$

To make use of Lemma 2.1, we observe that

$$
\left(I-\frac{z \sqrt{2 E}+2 I}{4}\right)^{\frac{1}{m}}=\sum_{k=0}^{\infty}(-1)^{k} C_{k}^{\frac{1}{m}}\left(\frac{z \sqrt{2 E}+2 I}{4}\right)^{k}=\sum_{k=0}^{\infty} \frac{(-1)^{k}}{2^{k}} C_{k}^{\frac{1}{m}}\left(\frac{z \sqrt{2 E}+2 I}{2}\right)^{k} .
$$

Therefore, we get

$$
\begin{aligned}
L_{\frac{1}{m}}(z, E) & =\frac{(-2)^{\frac{1}{m}}(\sqrt{2 E})^{-\frac{1}{m}}}{\Gamma\left(\frac{1}{m}+1\right) \cdot 2^{\frac{1}{m}}} \sum_{k=0}^{\infty} \frac{(-1)^{k}}{2^{2 k}} C_{k}^{\frac{1}{m}} D_{-1}^{\frac{1}{m}}(z \sqrt{2 E}+2 I)^{\frac{1}{m}+k} \\
& =(-1)^{k}(\sqrt{2 E})^{-\frac{1}{m}}(\sqrt{2 E})^{\frac{1}{m}} \sum_{k=0}^{\infty} \frac{\left(-\frac{1}{m}\right)_{k}\left(\frac{1}{m}+1\right)_{k}}{(\Gamma(k+1))^{2}}\left(\frac{2 I+z \sqrt{2 E}}{4}\right)^{k} \\
& =(-1)^{k} \sum_{k=0}^{\infty} \frac{\left(-\frac{1}{m}\right)_{k}\left(\frac{1}{m}+1\right)_{k}}{(1)_{k} k !}\left(\frac{2 I+z \sqrt{2 E}}{4}\right)^{k} \\
& =(-1)^{\frac{1}{m}}{ }_{2} F_{1}\left(-\frac{1}{m}, \frac{1}{m}+1 ; 1 ; \frac{z \sqrt{2 E}+2 I}{4}\right) .
\end{aligned}
$$


(iii) Similarly, in proof (ii), we obtain the required relationship.

\subsection{Property of continuity}

Theorem 3.2 If $m=1,2,3, \ldots$ and $\alpha \in(-1,0)$, then

(i) $\lim _{\frac{1}{m} \rightarrow 0^{+}} L_{\frac{1}{m}}(z, E)=\lim _{\alpha \rightarrow 0^{-}} L_{\alpha}(z, E)=1$.

(ii) $L_{\frac{1}{m}}(-z, E)=(-1)^{\frac{1}{m}} L_{\frac{1}{m}}(z, E)$.

Proof (i) According to relation (ii) in Theorem 3.1, we have

$$
\begin{aligned}
\lim _{\frac{1}{m} \rightarrow 0^{+}} L_{\frac{1}{m}}(z, E) & =\lim _{\frac{1}{m} \rightarrow 0^{+}}(-1)^{\frac{1}{m}}{ }_{2} F_{1}\left(-\frac{1}{m}, \frac{1}{m}+1 ; 1 ; \frac{2 I+z \sqrt{2 E}}{4}\right) \\
& =\lim _{\frac{1}{m} \rightarrow 0^{+}}(-1)^{\frac{1}{m}}\left\{\frac{\left(-\frac{1}{m}\right)_{0}\left(\frac{1}{m}+1\right)_{0}}{(1)_{0}}+\frac{\left(-\frac{1}{m}\right)_{1}\left(\frac{1}{m}+1\right)_{1}}{(1)_{1}} \frac{2 I+z \sqrt{2 E}}{4}+\cdots\right\},
\end{aligned}
$$

where $(\varsigma)_{0}=1,(0)_{s}=0$, then we obtain the required result as follows:

$$
\lim _{\frac{1}{m} \rightarrow 0^{+}} L_{\frac{1}{m}}(z, E)=1
$$

Similarly,

$$
\lim _{\alpha \rightarrow 0^{-}} L_{\alpha}(z, E)=1 .
$$

(ii) From relation (i) in Theorem 3.1, we can write

$$
\begin{aligned}
L_{\frac{1}{m}}(-z, E)= & \left\{\frac{1}{2}\left(\frac{-z \sqrt{2 E}}{2}-I\right)\right\}{ }^{\frac{1}{m}}{ }_{2} F_{1}\left(-\frac{1}{m},-\frac{1}{m} ; 1 ;\left(I-\frac{z \sqrt{2 E}}{2}\right)\left(\frac{-z \sqrt{2 E}}{2}-I\right)^{-1}\right) \\
= & (-1)^{\frac{1}{m}}\left\{\frac{1}{2}\left(\frac{z \sqrt{2 E}}{2}+I\right)\right\}^{\frac{1}{m}} \\
& \times{ }_{2} F_{1}\left(-\frac{1}{m},-\frac{1}{m} ; 1 ;\left(\frac{z \sqrt{2 E}}{2}-I\right)\left(\frac{z \sqrt{2 E}}{2}+I\right)^{-1}\right) .
\end{aligned}
$$

Thus,

$$
L_{\frac{1}{m}}(-z, E)=(-1)^{\frac{1}{m}} L_{\frac{1}{m}}(z, E)
$$

\section{Recurrence relations and matrix differential equations}

Two main results are given in this section. The first one is concerned with the Legendretype matrix polynomials of arbitrary fractional orders $L_{\frac{1}{m}}(z, E)$, which generalizes its counterpart given in [36]. The second main result provides a differential equation related to $L_{\frac{1}{m}}(z, E)$.

Theorem 4.1 The matrix polynomials $L_{\frac{1}{m}}(z, E)$ have the following recurrence relations:

(i) $2 L_{\frac{1}{m}+1}^{\prime}(z, E)-2 L_{\frac{1}{m}-1}^{\prime}(z, E)=\left(\frac{2}{m}+1\right) \sqrt{2 E} L_{\frac{1}{m}}(z, E)$.

(ii) $2 L_{\frac{1}{m}+1}^{\prime}(z, E)=\left(\frac{1}{m}+1\right) \sqrt{2 E} L_{\frac{1}{m}}(z, E)+z \sqrt{2 E} L_{\frac{1}{m}}^{\prime}(z, E)$. 
(iii) $z \sqrt{2 E} L_{\frac{1}{m}}^{\prime}(z, E)-\frac{1}{m} \sqrt{2 E} L_{\frac{1}{m}}(z, E)=2 L_{\frac{1}{m}-1}^{\prime}(z, E)$.

Proof (i) Since

$$
L_{\frac{1}{m}}(z, E)=\frac{1}{\Gamma\left(\frac{1}{m}+1\right)} D_{-1}^{\frac{1}{m}}(\sqrt{2 E})^{-\frac{1}{m}}\left\{\left(\frac{z \sqrt{2 E}}{2}\right)^{2}-I\right\}^{\frac{1}{m}},
$$

then

$$
L_{\frac{1}{m}+1}(z, E)=\frac{1}{\Gamma\left(\frac{1}{m}+2\right)} D_{-1}^{\frac{1}{m}+1}(\sqrt{2 E})^{-\left(\frac{1}{m}+1\right)}\left\{\left(\frac{z \sqrt{2 E}}{2}\right)^{2}-I\right\}^{\frac{1}{m}+1} .
$$

Hence, it follows

$$
\begin{aligned}
L_{\frac{1}{m}+1}(z, E)= & \frac{(\sqrt{2 E})^{-\left(\frac{1}{m}+1\right)}}{\Gamma\left(\frac{1}{m}+2\right)}\left\{I_{-1}^{1-\frac{1}{m}} D^{2}\left(\left(\frac{z \sqrt{2 E}}{2}\right)^{2}-I\right)^{\frac{1}{m}+1}\right\} \\
= & \frac{(\sqrt{2 E})^{-\left(\frac{1}{m}+1\right)}}{\Gamma\left(\frac{1}{m}+2\right)}\left\{I_{-1}^{1-\frac{1}{m}} D\left[\left(\frac{1}{m}+1\right)\left(\left(\frac{z \sqrt{2 E}}{2}\right)^{2}-I\right)^{\frac{1}{m}} \frac{2 z(\sqrt{2 E})^{2}}{4}\right]\right\} \\
= & \frac{(\sqrt{2 E})^{1-\frac{1}{m}}}{2 \Gamma\left(\frac{1}{m}+1\right)} \\
& \times\left\{I_{-1}^{1-\frac{1}{m}}\left[\left(\left(\frac{z \sqrt{2 E}}{2}\right)^{2}-I\right)^{\frac{1}{m}}+\frac{2(z \sqrt{2 E})^{2}}{4 m}\left(\left(\frac{z \sqrt{2 E}}{2}\right)^{2}-I\right)^{\frac{1}{m}-1}\right]\right\} \\
= & \frac{(\sqrt{2 E})^{1-\frac{1}{m}}}{2 \Gamma\left(\frac{1}{m}+1\right)} \\
& \times\left\{I_{-1}^{1-\frac{1}{m}}\left[\left(\frac{2}{m}+1\right)\left(\left(\frac{z \sqrt{2 E}}{2}\right)^{2}-I\right)^{\frac{1}{m}}+\frac{2}{m}\left(\left(\frac{z \sqrt{2 E}}{2}\right)^{2}-I\right)^{\frac{1}{m}-1}\right]\right\} .
\end{aligned}
$$

We now have

$$
\begin{aligned}
L_{\frac{1}{m}+1}(z, E)= & \frac{(\sqrt{2 E})^{1-\frac{1}{m}}}{2 \Gamma\left(\frac{1}{m}+1\right)} I_{-1}^{1-\frac{1}{m}}\left\{\left(\frac{2}{m}+1\right)\left(\left(\frac{z \sqrt{2 E}}{2}\right)^{2}-I\right)^{\frac{1}{m}}\right\} \\
& +\frac{(\sqrt{2 E})^{1-\frac{1}{m}}}{\Gamma\left(\frac{1}{m}\right)} I_{-1}^{1-\frac{1}{m}}\left(\left(\frac{z \sqrt{2 E}}{2}\right)^{2}-I\right)^{\frac{1}{m}-1} .
\end{aligned}
$$

Differentiating both sides, we get

$$
\begin{aligned}
L_{\frac{1}{m}+1}^{\prime}(z, E)= & \left(\frac{2}{m}+1\right)\left(\frac{\sqrt{2 E}}{2}\right) \frac{D_{-1}^{\frac{1}{m}}(\sqrt{2 E})^{-\frac{1}{m}}}{\Gamma\left(\frac{1}{m}+1\right)}\left\{\left(\frac{x \sqrt{2 A}}{2}\right)^{2}-I\right\}^{\frac{1}{m}} \\
& +\frac{D D_{-1}^{\frac{1}{m}-1}(\sqrt{2 E})^{-\left(\frac{1}{m}-1\right)}}{\Gamma\left(\frac{1}{m}\right)}\left\{\left(\frac{z \sqrt{2 E}}{2}\right)^{2}-I\right\}^{\frac{1}{m}-1} \\
= & \left(\frac{2}{m}+1\right)\left(\frac{\sqrt{2 E}}{2}\right) L_{\frac{1}{m}}(z, E)+L_{\frac{1}{m}-1}^{\prime}(z, E) .
\end{aligned}
$$

Thus the proof of (i) in Theorem 4.1 is completed. 
(ii) Also we have

$$
\begin{aligned}
L_{\frac{1}{m}+1}(z, E) & =\frac{D_{-1}^{\frac{1}{m}+1}(\sqrt{2 E})^{-\left(\frac{1}{m}+1\right)}}{\Gamma\left(\frac{1}{m}+2\right)}\left\{\left(\frac{z \sqrt{2 E}}{2}\right)^{2}-I\right\}^{\frac{1}{m}+1} \\
& =\frac{(\sqrt{2 E})^{-\left(\frac{1}{m}+1\right)}}{\Gamma\left(\frac{1}{m}+2\right)} D_{-1}^{\frac{1}{m}} D\left\{\left(\frac{z \sqrt{2 E}}{2}\right)^{2}-I\right\}^{\frac{1}{m}+1} \\
& =\frac{2\left(\frac{1}{m}+1\right)(\sqrt{2 E})^{-\left(\frac{1}{m}+1\right)}(\sqrt{2 E})^{2}}{4 \Gamma\left(\frac{1}{m}+2\right)} D_{-1}^{\frac{1}{m}}\left\{z\left(\left(\frac{z \sqrt{2 E}}{2}\right)^{2}-I\right)^{\frac{1}{m}}\right\} \\
& =\frac{(\sqrt{2 E})^{1-\frac{1}{m}}}{2 \Gamma\left(\frac{1}{m}+1\right)} D_{-1}^{\frac{1}{m}}\left\{z\left(\left(\frac{z \sqrt{2 E}}{2}\right)^{2}-I\right)^{\frac{1}{m}}\right\} .
\end{aligned}
$$

Using the Leibniz rule for fractional derivative, we have

$$
\begin{aligned}
L_{\frac{1}{m}+1}(z, E) & =\frac{(\sqrt{2 E})^{1-\frac{1}{m}}}{2 \Gamma\left(\frac{1}{m}+1\right)}\left\{\sum_{k=0}^{\infty} C_{k}^{\frac{1}{m}} D^{k} x D_{-1}^{\frac{1}{m}-k}\left(\left(\frac{z \sqrt{2 E}}{2}\right)^{2}-I\right)^{\frac{1}{m}}\right\} \\
& =\frac{(\sqrt{2 E})^{1-\frac{1}{m}}}{2 \Gamma\left(\frac{1}{m}+1\right)}\left\{z D_{-1}^{\frac{1}{m}}\left(\left(\frac{z \sqrt{2 E}}{2}\right)^{2}-I\right)^{\frac{1}{m}}+\frac{1}{m} D_{-1}^{\frac{1}{m}-1}\left(\left(\frac{z \sqrt{2 E}}{2}\right)^{2}-I\right)^{\frac{1}{m}}\right\} .
\end{aligned}
$$

Multiplying it by 2 and differentiating it, we obtain

$$
\begin{aligned}
2 L_{\frac{1}{m}+1}^{\prime}(z, E) & =\sqrt{2 E}\left\{z L_{\frac{1}{m}}^{\prime}(z, E)+L_{\frac{1}{m}}(z, E)+\frac{1}{m} L_{\frac{1}{m}}(z, E)\right\} \\
& =\left(\frac{1}{m}+1\right) \sqrt{2 E} L_{\frac{1}{m}}(z, E)+z \sqrt{2 E} L_{\frac{1}{m}}^{\prime}(z, E),
\end{aligned}
$$

which is the required result in (ii).

(iii) Subtracting (i) and (ii), we get

$$
z \sqrt{2 E} L_{\frac{1}{m}}^{\prime}(z, E)-\frac{1}{m} \sqrt{2 E} L_{\frac{1}{m}}(z, E)=2 L_{\frac{1}{m}-1}^{\prime}(z, E),
$$

which is the required result in (iii).

Theorem 4.2 For each natural number $m \geq 1$, the Legendre-type matrix polynomials $L_{\frac{1}{m}}(z, E)$ will satisfy the following matrix differential equation:

$$
\begin{aligned}
& \left(4 I-(z \sqrt{2 E})^{2}\right) L_{\frac{1}{m}}^{\prime \prime}(z, E)-2 x(\sqrt{2 E})^{2} L_{\frac{1}{m}}^{\prime}(z, E) \\
& +\frac{1}{m}\left(\frac{1}{m}+1\right)(\sqrt{2 E})^{2} L_{\frac{1}{m}}(z, E)=\mathbf{0}, \quad|z| \leq 1 .
\end{aligned}
$$

Proof From (ii) in Theorem 4.1, we have

$$
2 L_{\frac{1}{m}+1}^{\prime}(z, E)=\left(\frac{1}{m}+1\right) \sqrt{2 E} L_{\frac{1}{m}}(z, E)+z \sqrt{2 E} L_{\frac{1}{m}}^{\prime}(z, E) .
$$


Replacing $\frac{1}{m}$ by $\frac{1}{m}-1$ to get

$$
2 L_{\frac{1}{m}}^{\prime}(z, E)=\frac{1}{m} \sqrt{2 E} L_{\frac{1}{m}-1}(z, E)+z \sqrt{2 E} L_{\frac{1}{m}-1}^{\prime}(z, E) .
$$

Differentiating (18) with respect to $z$, we have

$$
2 L_{\frac{1}{m}}^{\prime \prime}(z, E)=\frac{1}{m} \sqrt{2 E} L_{\frac{1}{m}-1}^{\prime}(z, E)+\sqrt{2 E} L_{\frac{1}{m}-1}^{\prime}(z, E)+z \sqrt{2 E} L_{\frac{1}{m}-1}^{\prime \prime}(z, E),
$$

thus

$$
2 L_{\frac{1}{m}}^{\prime \prime}(z, E)=\left(\frac{1}{m}+1\right) \sqrt{2 E} L_{\frac{1}{m}-1}^{\prime}(z, E)+z \sqrt{2 E} L_{\frac{1}{m}-1}^{\prime \prime}(z, E) .
$$

Also, from (iii) in Theorem 4.1, we have

$$
z \sqrt{2 E} L_{\frac{1}{m}}^{\prime}(z, E)-\frac{1}{m} \sqrt{2 E} L_{\frac{1}{m}}(z, E)=2 L_{\frac{1}{m}-1}^{\prime}(z, E) .
$$

Differentiating (21) with respect to $z$, we have

$$
\sqrt{2 E} L_{\frac{1}{m}}^{\prime}(z, E)+z \sqrt{2 E} L_{\frac{1}{m}}^{\prime \prime}(z, E)-\frac{1}{m} \sqrt{2 E} L_{\frac{1}{m}}^{\prime}(z, E)=2 L_{\frac{1}{m}-1}^{\prime \prime}(z, E),
$$

thus

$$
\left(1-\frac{1}{m}\right) \sqrt{2 E} L_{\frac{1}{m}}^{\prime}(z, E)+x \sqrt{2 E} L_{\frac{1}{m}}^{\prime \prime}(z, E)=2 L_{\frac{1}{m}-1}^{\prime \prime}(z, E) .
$$

Multiplying (22) by 2, we get

$$
4 L_{\frac{1}{m}}^{\prime \prime}(z, E)=2\left(\frac{1}{m}+1\right) \sqrt{2 E} L_{\frac{1}{m}-1}^{\prime}(z, E)+2 z \sqrt{2 E} L_{\frac{1}{m}-1}^{\prime \prime}(z, E) .
$$

From (21) and (23) in (24), we obtain

$$
\begin{aligned}
4 L_{\frac{1}{m}}^{\prime \prime}(z, E)= & \left(\frac{1}{m}+1\right) \sqrt{2 E}\left\{z \sqrt{2 E} L_{\frac{1}{m}}^{\prime}(z, E)-\frac{1}{m} \sqrt{2 E} L_{\frac{1}{m}}(z, E)\right\} \\
& +x \sqrt{2 E}\left\{\left(1-\frac{1}{m}\right) \sqrt{2 E} L_{\frac{1}{m}}^{\prime}(z, E)+z \sqrt{2 E} L_{\frac{1}{m}}^{\prime \prime}(z, E)\right\} .
\end{aligned}
$$

Therefore, the proof Theorem 4.2 is completed.

Corollary 4.1 For $m=1,2,3, \ldots, W(\cos \phi, E)=L_{\frac{1}{m}}(\cos \phi, E)$ satisfying the following differential equation:

$$
\frac{(\sqrt{2 E})^{2}}{\sin \phi} \frac{d}{d \phi}\left(\sin \phi \frac{d W}{d \phi}\right)+\frac{1}{m}\left(\frac{1}{m}+1\right)(\sqrt{2 E})^{2} W=\mathbf{0} .
$$

Proof Replacing $\frac{z \sqrt{2 E}}{2}$ by $I \cos \phi$ in equation (18), we have the required result. 


\section{Laplace's first integral form and orthogonality property}

Two more basic properties of the Legendre-type matrix polynomials of arbitrary fractional orders $L_{\frac{1}{m}}(z, E)$ are developed in this section. That they enjoy an integral form is obtained in Theorem 5.1 in Sect. 5.1 through exploitation of their Definition 3.1 in terms of the hypergeometric matrix function. Orthogonality as an integral over $[-1,1]$ is also the subject of Theorem 5.2 in Sect. 5.2.

\subsection{Laplace's first integral form}

Theorem 5.1 The Legendre-type matrix polynomials of arbitrary (fractional) orders $L_{\frac{1}{m}}(z, E)$ have the following Laplace's first integral form:

$$
L_{\frac{1}{m}}(z, E)=\frac{1}{\pi} \int_{0}^{\pi}\left[\frac{z \sqrt{2 E}}{2}+\left(\left(\frac{z \sqrt{2 E}}{2}\right)^{2}-I\right)^{\frac{1}{2}} \cos \varphi\right]^{\frac{1}{m}} d \varphi .
$$

Proof According to Theorem 3.2, we can write

$$
\begin{aligned}
L_{\frac{1}{m}}(z, E) & =(-1)^{\frac{1}{m}}{ }_{2} F_{1}\left(-\frac{1}{m}, \frac{1}{m}+1 ; 1 ; \frac{2 I+z \sqrt{2 E}}{4}\right) \\
& ={ }_{2} F_{1}\left(-\frac{1}{m}, \frac{1}{m}+1 ; 1 ; \frac{2 I-z \sqrt{2 E}}{4}\right) \\
& ={ }_{2} F_{1}\left(-\frac{1}{m}, \frac{1}{m}+1 ; 1 ; \frac{I-\frac{z \sqrt{2 E}}{2}}{2}\right) .
\end{aligned}
$$

Therefore, we obtain

$$
\begin{aligned}
L_{\frac{1}{m}}(z, E) & ={ }_{2} F_{1}\left(-\frac{1}{m}, \frac{1}{m}+1 ; 1 ; \frac{I-\frac{z \sqrt{2 E}}{2}}{2}\right) \\
& =\left(\frac{z \sqrt{2 E}}{2}\right)^{\frac{1}{m}}{ }_{2} F_{1}\left(-\frac{1}{2 m},-\frac{1}{2 m}+\frac{1}{2} ; 1 ;\left\{\left(\left(\frac{z \sqrt{2 E}}{2}\right)^{2}-I\right)\left(\left(\frac{z \sqrt{2 E}}{2}\right)^{-1}\right)^{2}\right\}\right) \\
& =\left(\frac{z \sqrt{2 E}}{2}\right)^{\frac{1}{m}} \sum_{k=0}^{\infty} \frac{\left(-\frac{1}{2 m}\right)_{k}\left(-\frac{1}{2 m}+\frac{1}{2}\right)_{k}}{(1)_{k} k !}\left\{\left(\left(\frac{z \sqrt{2 E}}{2}\right)^{2}-I\right)\left(\left(\frac{z \sqrt{2 E}}{2}\right)^{-1}\right)^{2}\right\}^{k} .
\end{aligned}
$$

Note that

$$
\left(-\frac{1}{2 m}\right)_{k}\left(-\frac{1}{2 m}+\frac{1}{2}\right)_{k}=\frac{\left(-\frac{1}{m}\right)_{2 k}}{2^{2 k}}=\frac{(-1)^{2 k} \Gamma\left(\frac{1}{m}+1\right)}{2^{2 k} \Gamma\left(\frac{1}{m}-2 k+1\right)} .
$$

Now, we see that

$$
L_{\frac{1}{m}}(z, E)=\sum_{k=0}^{\infty}\left\{\frac{\Gamma\left(\frac{1}{m}+1\right)\left(\frac{z \sqrt{2 E}}{2}\right)^{\frac{1}{m}-2 k}\left(\left(\frac{z \sqrt{2 E}}{2}\right)^{2}-I\right)^{k}}{2^{2 k}(k !)^{2} \Gamma\left(\frac{1}{m}-2 k+1\right)}\right\}
$$

where $\left(\frac{1}{2}\right)_{k}=\frac{\Gamma(2 k+1)}{2^{2 k} k !}=\frac{(2 k) !}{2^{2 k} k !} \Rightarrow 2^{2 k} k !=\frac{(2 k) !}{\left(\frac{1}{2}\right)_{k}}$, then

$$
L_{\frac{1}{m}}(z, E)=\sum_{k=0}^{\infty}\left\{\frac{\Gamma\left(\frac{1}{m}+1\right)\left(\frac{1}{2}\right)_{k}\left(\frac{z \sqrt{2 E}}{2}\right)^{\frac{1}{m}-2 k}\left(\left(\frac{z \sqrt{2 E}}{2}\right)^{2}-I\right)^{k}}{k !(2 k) ! \Gamma\left(\frac{1}{m}-2 k+1\right)}\right\} .
$$


But we have $\frac{\left(\frac{1}{2}\right)_{k}}{k !}=\frac{1}{\pi} \int_{0}^{\pi} \cos ^{2 k} \varphi d \varphi$ and $\int_{0}^{\pi} \cos ^{s} \varphi d \varphi=0$ for odd $s$. Hence,

$$
L_{\frac{1}{m}}(z, E)=\frac{1}{\pi} \sum_{k=0}^{\infty}\left\{\frac{\Gamma\left(\frac{1}{m}+1\right)\left(\frac{z \sqrt{2 E}}{2}\right)^{\frac{1}{m}-k}\left(\left(\frac{z \sqrt{2 E}}{2}\right)^{2}-I\right)^{\frac{1}{2} k}}{k ! \Gamma\left(\frac{1}{m}-k+1\right)}\right\} \int_{0}^{\pi} \cos ^{k} \varphi d \varphi,
$$

in which each term involving an odd $k$ is zero, yields the desired result of Theorem 5.1.

\subsection{Orthogonality property}

Theorem 5.2 Let $\mu, v=1,2,3, \ldots$. Then the Legendre-type matrix polynomials of arbitrary (fractional) orders $L_{\frac{1}{\mu}}(z, E)$ and $L_{\frac{1}{v}}(z, E)$ satisfy the following property:

$$
\int_{-1}^{1} L_{\frac{1}{\mu}}(z, E) L_{\frac{1}{v}}(z, E) d z=0 ; \quad \mu \neq v
$$

Proof From Theorem 4.2, for any two numbers $\mu \neq \nu$, we have

$$
\left(4 I-(z \sqrt{2 E})^{2}\right) L_{\frac{1}{\mu}}^{\prime \prime}(z, E)-2 z(\sqrt{2 E})^{2} L_{\frac{1}{\mu}}^{\prime}(z, E)+\frac{1}{\mu}\left(\frac{1}{\mu}+1\right)(\sqrt{2 E})^{2} L_{\frac{1}{\mu}}(z, E)=\mathbf{0}
$$

and

$$
\left(4 I-(z \sqrt{2 E})^{2}\right) L_{\frac{1}{v}}^{\prime \prime}(z, E)-2 z(\sqrt{2 E})^{2} L_{\frac{1}{v}}^{\prime}(z, E)+\frac{1}{v}\left(\frac{1}{v}+1\right)(\sqrt{2 E})^{2} L_{\frac{1}{v}}(z, E)=\mathbf{0} .
$$

By multiplying (33) by $L_{\frac{1}{v}}(z, E)$ and (34) by $L_{\frac{1}{\mu}}(z, E)$, subtracting the resulting equations, we have

$$
\begin{aligned}
(4 I- & \left.(z \sqrt{2 E})^{2}\right)\left[L_{\frac{1}{\mu}}^{\prime \prime}(z, E) L_{\frac{1}{v}}(z, E)-L_{\frac{1}{v}}^{\prime \prime}(z, E) L_{\frac{1}{\mu}}(z, E)\right] \\
& -2 z(\sqrt{2 E})^{2}\left[L_{\frac{1}{\mu}}^{\prime}(z, E) L_{\frac{1}{v}}(z, E)-L_{\frac{1}{v}}^{\prime}(z, E) L_{\frac{1}{\mu}}(z, E)\right] \\
& +(\sqrt{2 E})^{2}\left[\frac{1}{\mu}\left(\frac{1}{\mu}+1\right)-\frac{1}{v}\left(\frac{1}{v}+1\right)\right] P_{\frac{1}{\mu}}(z, E) L_{\frac{1}{v}}(z, E)=\mathbf{0} \\
= & \frac{d}{d z}\left(4 I-(z \sqrt{2 E})^{2}\right)\left[L_{\frac{1}{\mu}}^{\prime}(z, E) L_{\frac{1}{v}}(z, E)-L_{\frac{1}{v}}^{\prime}(z, E) L_{\frac{1}{\mu}}(z, E)\right] \\
& +(\sqrt{2 E})^{2}\left[\frac{1}{\mu}\left(\frac{1}{v}+1\right)-\frac{1}{v}\left(\frac{1}{v}+1\right)\right] L_{\frac{1}{\mu}}(z, E) L_{\frac{1}{v}}(z, E)=\mathbf{0} .
\end{aligned}
$$

Integrating from -1 to 1 , we obtain the result in (32).

\section{Conclusion}

The Legendre polynomials $\mathbb{P}_{n}(z)$ are given by the following Rodrigues' formula [41]:

$$
\mathbb{P}_{n}(z)=\frac{1}{2^{n} n !} \frac{d^{n}}{d z^{n}}\left(z^{2}-1\right)^{n}, \quad n \in \mathbb{N}_{0} .
$$

These polynomials satisfy the second order linear differential equation

$$
\frac{d}{d z}\left[\left(1-z^{2}\right) \frac{d \mathbb{P}_{n}(z)}{d z}\right]+n(n+1) \mathbb{P}_{n}(z)=0 ; \quad|z| \leq 1 .
$$


Legendre polynomial is an important orthogonal polynomial with interval of orthogonality between -1 and 1 , in the form

$$
\int_{-1}^{1} \mathbb{P}_{m}(z) \mathbb{P}_{n}(z) d z= \begin{cases}0 & \text { if } n \neq m \\ \frac{2}{2 n+1} & \text { if } m=n\end{cases}
$$

Because of their orthogonal properties, Legendre polynomials have been used for solving other integral equations such as Fredholm integral equations [31], Bagley-Torvik equations [33], and Volterra integral equations [34]. Recently, orthogonal matrix polynomials and their generalizations in different ways have been a focus of increasing attention leading to new and interesting problems. In this perspective, this manuscript is a continuation of the recent authors' paper [1], where we have discussed the Legendre-type matrix polynomials via fractional orders, starting from the Rodrigues matrix formula. This study is assumed to be a generalization of the scalar case [39] to the matrix setting. This approach allows us to derive several new integral and differential representations that can be used in theoretical and applicable aspects and for some numerical methods.

\section{Acknowledgements}

The authors extend their appreciation to the Deanship of Scientific Research at King Khalid University for funding this work through research groups program under grant (R.G.P.1/184/41). The authors also would like to thank Prof. N. Saad for his valuable suggestions and comments to improve this paper.

Funding

Deanship of Scientific Research at King Khalid University.

Availability of data and materials

The data that support the findings of this study are available from the authors, upon request.

Competing interests

The authors declare that they have no competing interests.

Authors' contributions

All authors jointly worked on the results and they read and approved the final manuscript.

\section{Author details}

'Department of Mathematics, College of Science, King Khalid University, P.O. Box 9004, 61413, Abha, Saudi Arabia.

${ }^{2}$ Department of Mathematics, Faculty of Science, South Valley University, 83523 Qena, Egypt. ${ }^{3}$ Mathematics Department, Faculty of Science, Sohag University, Sohag, 82524 Sohag, Egypt.

\section{Publisher's Note}

Springer Nature remains neutral with regard to jurisdictional claims in published maps and institutional affiliations.

Received: 1 May 2020 Accepted: 14 September 2020 Published online: 18 September 2020

\section{References}

1. Zayed, M., Abul-Ez, M., Abdalla, M., Saad, N.: On the fractional order Rodrigues formula for the shifted Legendre-type matrix polynomials. Mathematics 8, 136 (2020)

2. Agarwal, P., Baleanu, D., Chen, Y., Momani, S., Machado, J.: Fractional Calculus: ICFDA 2018, Amman, Jordan, July 16-18. Springer Proceedings in Mathematics Statistics, vol. 303 (2020)

3. Agarwal, P., Jain, S., Mansour, T.: Further extended Caputo fractional derivative operator and its applications. Russ. J. Math. Phys. 24, 415-425 (2017)

4. Agarwal, P.: Some inequalities involving Hadamard type $k$-fractional integral operators. Math. Methods Appl. Sci. 40, 3882-3891 (2017)

5. Zhang, $X$ :: The non-uniqueness of solution for initial value problem of impulsive differential equations involving higher order Katugampola fractional derivative. Adv. Differ. Equ. 2020, 85 (2020)

6. Shiri, B., Baleanu, D.: System of fractional differential algebraic equations with applications. Chaos Solitons Fractals 120, 203-212 (2019)

7. Srivastava, H., Abbas, S., Tyagi, S., Lassoued, D.: Global exponential stability of fractional-order impulsive neural network with time-varying and distributed delay. Math. Methods Appl. Sci. 37, 1-10 (2018)

8. Kiryakova, V.: All the special functions are fractional differ-integrals of elementary functions. J. Phys. A, Math. Gen. 30 5085-5103 (1997) 
9. Kiryakova, V.: The special functions of fractional calculus as generalized fractional calculus operators of some basic functions. Comput. Math. Appl. 59, 1128-1141 (2010)

10. Kiryakova, V:: Fractional calculus operators of special functions? The result is well predictable. Chaos Solitons Fractals $102,2-15(2017)$

11. Kiryakova, V:: Use of fractional calculus to evaluate some improper integrals of special functions. AIP Conf. Proc. 1910, 050012 (2017). https://doi.org/10.1063/1.5013994

12. Agarwal, P., Agarwal, R., Ruzhansky, M.: Special Functions and Analysis of Differential Equations, 1st edn. CRC Press, Boca Raton (2020)

13. Agarwal, P., Al-Mdallal, Q., Je Cho, Y., Jain, S.: Fractional differential equations for the generalized Mittag-Leffler function. Adv. Differ. Equ. 2018, 58 (2018)

14. Jain, S., Agarwal, P., Kilicman, A.: Pathway fractional integral operator associated with 3m-parametric Mittag-Leffler functions. Int. J. Appl. Comput. Math. 4, 115 (2018)

15. Agarwal, R., Jain, S., Agarwal, R.P., Baleanu, D.: A remark on the fractional integral operators and the image formulas of generalized Lommel-Wright function. Front. Phys. 6, 79 (2018)

16. Mathai, A., Haubold, H.: An Introduction to Fractional Calculus. Nova Science Publishers, New York (2017). ISBN: 9781536120424

17. Bakhet, A., He, F.: On 2-variables Konhauser matrix polynomials and their fractional integrals. Mathematics 8, 232 (2020)

18. Bakhet, A., Jiao, Y., He, F.: On the Wright hypergeometric matrix functions and their fractional calculus. Integral Transforms Spec. Funct. 30, 138-156 (2019)

19. Abdalla, M.: Fractional operators for the Wright hypergeometric matrix functions. Adv. Differ. Equ. 2020, 246 (2020)

20. Abdalla, M., Hidan, M.: Fractional orders of the generalized Bessel matrix polynomials. Eur. J. Pure Appl. Math. 10, 995-1004 (2017)

21. Defez, E.: A Rodrigues-type formula for Gegenbauer matrix polynomials. Appl. Math. Lett. 26, 899-903 (2013)

22. Abdalla, M.: Operational formula for the generalized Bessel matrix polynomials. J. Mod. Methods Numer. Math. 8, 156-163 (2017)

23. Abdalla, M., Abd-Elmageed, H., Abul-Ez, M., Kishka, Z:: Operational formulae of the multivariable hypergeometric matrix functions and related matrix polynomials. Gen. Lett. Math. 3, 81-90 (2017)

24. Abdalla, M.: Further results on the generalised hypergeometric matrix functions. Int. J. Comput. Sci. Math. 10, 1-10 (2019)

25. Abdalla, M.: Special matrix functions: characteristics, achievements and future directions. Linear Multilinear Algebra $68,1-28(2020)$

26. Dicke, R., Wittke, J.: Introduction to Quantum Mechanics. Addison-Wesley, Reading (1960)

27. Hollas, J.: Modern Spectroscopy. Wiley, Chichester (1992)

28. Paterson, R.: A First Course in Fluid Dynamics. Cambridge University Press, Cambridge (1983)

29. Liao, X., Zhang, K.: A new Legendre-type polynomial and its application to geostrophic flow in rotating fluid spheres. Proc. R. Soc. A 466, 2203-2217 (2010)

30. Brown, G., Kouniandos, S., Wang, K.: On the positivity of some basic Legendre polynomial sums. J. Lond. Math. Soc. 59, 939-954 (1999)

31. Maleknejad, K., Nouri, K., Yousefi, M.: Discussion on convergence of Legendre polynomial for numerical solution of integral equations. Appl. Math. Comput. 193, 335-339 (2007)

32. Anli, F., Gungor, S.: Some useful properties of Legendre polynomials and its applications to neutron transport equation in slab geometry. Appl. Math. Model. 31, 727-733 (2007)

33. Rahimkhani, P., Ordokhani, Y.: Application of Muntz-Legendre polynomials for solving the Bagley-Torvik equation in a large interval. SeMA J. 75, 517-533 (2018)

34. Liu, Y.: Application of Legendre polynomials in solving Volterra integral equations of the second kind. Appl. Math. 3, 157-159 (2013)

35. Abedian, A., Duster, A.: Equivalent Legendre polynomials: numerical integration of discontinuous functions in the finite element methods. Comput. Methods Appl. Mech. Eng. 343, 690-720 (2019)

36. Upadhyaya, L., Shehata, A.: On Legendre matrix polynomials and its applications. I. Trans. Math. Sci. Comput. 4, 291-310 (2011)

37. Shehata, A.: A new kind of Legendre matrix polynomials. Gazi Univ. J. Sci. 26, 435-457 (2016)

38. Lancaster, P.: Theory of Matrices. Academic Press, New York (1969)

39. Rida, S., Yousef, A.: On the fractional order Rodrigues formula for the Legendre polynomials $P_{\frac{1}{n}}(x)$. Adv. Appl. Math. Sci. 10, 509-517 (2011)

40. Jódar, L., Company, R.: Hermite matrix polynomials and second order matrix differential equations. J. Approx. Theory Appl. 12, 20-30 (1996)

41. Attar, R.: Special Functions and Orthogonal Polynomials. Lulu Press, Morrisville (2006) 